\title{
LOS BIOCARBURANTES SOSTENIBLES: UN ANÁLISIS DEL MARCO REGULATORIO DE LA UNIÓN EUROPEA
}

\author{
Izabel Rigo Portocarrero ${ }^{1}$ \\ Universidad de Salamanca (USAL) \\ Daniel Amin Ferraz ${ }^{2}$ \\ Centro Universitário de Brasília (UniCEUB) \\ Liziane Paixão Silva Oliveira ${ }^{3}$ \\ Universidade Tiradentes (UNIT)
}

\section{RESUMEN}

Los recursos naturales son finitos, y la intensidad de la contaminación establecida en la sociedad moderna es insostenible. No se debe seguir sobrecargando la biosfera sin antes considerar sus límites y replantear los patrones de consumo y utilización de los recursos naturales. Con base en tal escenario, se fijaron metas muy ambiciosas relativas al desarrollo de combustibles alternativos. En particular, en la Unión Europea (UE), se estableció un importante objetivo de sustitución de un $10 \%$ de combustibles fósiles por fuentes renovables en el sector de los transportes para 2020. Sin embargo, surgieron dudas sobre la sostenibilidad de la expansión de la demanda de la primera generación de esta energía, que aumenta la presión para intensificar la producción agrícola, generando una potencial amenaza a la seguridad alimentaria. En la UE, estas preocupaciones se tradujeron en una fuerte oposición a la producción de la primera generación de biocarburantes y el incentivo a un desarrollo cauteloso de las generaciones más avanzadas, orientando el enfoque de la política europea de biocarburantes hacia la sostenibilidad. En este contexto, se propone analizar la evolución

1 Doctora en Derecho por la USAL. Programa de Doctorado en Estado de Derecho y Gobernanza Global. Departamento de Economía Aplicada. Facultad de Derecho. ORCID: https://orcid.org/00000002-2267-9902 / e-mail: izabelrigo@gmail.com

2 Doutor em Direito Internacional pela Universidad de València (UV). Mestre em Direito Empresarial na Universidade de Coimbra (UC). Bacharel em Direito pela Universidade Federal de Juiz de Fora (UFJF). Professor titular em Direito Empresarial no PPGD do UniCEUB. Advogado. E-mail: daniel. amin@afcadvogados.adv.br

3 Estágio Pós-doutoral na Universidade de Brasília (UnB), com bolsa da Fapitec-SE/CAPES. Doutora em Direito Internacional Público pela Université Aix Marseille III. Mestre em Direito pela UnB. Especialista em Direito Ambiental pelo Centro Universitário de Brasília (UniCEUB). Bacharel em Direito pela UNIT. Professora titular na UNIT. Professora na Universidade de Vila Velha (UVV). Advogada. ORCID: https://orcid.org/0000-0002-6266-6073 / e-mail: lizianeoliveira1@yahoo.com.br 
de las regulaciones europeas para los biocombustibles sostenibles entre 2005-2014, etapa que coincide con la madurez de la producción y el consumo global de los biocarburantes, además de abarcar los cinco años previos y posteriores a la entrada en vigor de la Directiva de Energías Renovables de la UE. Este estudio se realizó con la revisión bibliográfica de fuentes primarias y secundarias, basada en textos normativos de la Unión Europea.

Palabras-clave: biocarburantes; sostenibilidad; Unión Europea.

\section{SUSTAINABLE BIOFUELS: AN ANALYSIS OF THE REGULATORY FRAMEWORK OF EUROPEAN UNION}

\section{ABSTRACT}

Natural resources are finite. In addition, it is necessary to mention the non-sustainability of exploitation of the same natural resources and the contamination of the environment. We should not continue to overburden the biosphere without considering its limits, rediscussing consumption patterns and the use of natural resources. Based on this scenario, were set ambitious targets for the development of alternative fuels. Thus, the European Union (EU) has established the objective of replacing $10 \%$ of fossil fuels with renewable sources in the transport sector, up to 2020. However, doubts arose about the sustainability of the demand expansion of the first generation of this energy, since it intensifies the agricultural production, generating potential threat to food security. In the EU these concerns have resulted in strong opposition to the production of the first generation of biocarburants, generating the need for a focus on European biofuels policy towards sustainability. In this context, the text proposes to analyze the evolution of European regulation for sustainable biocarburants between 2005-2014, a period that coincides with the maturity of the production and global consumption of biofuels, in addition to covering the five years before and after the entry into force of the EU Renewable Energy Directive. This study was conducted based on a bibliographic review of primary and secondary sources, with emphasis on the European Union's normative texts and reports.

Keywords: biofuels; European Union; susteinability. 


\section{INTRODUCCIÓN}

En un mundo donde los recursos naturales son finitos y los sumideros ambientales tienen una capacidad de absorción restringida, la intensidad de explotación y contaminación establecida en la sociedad moderna es insostenible. Cuando se camina en el sentido de agotar un recurso indispensable, como son los combustibles fósiles, o se sobrecarga la capacidad de absorción de los contaminantes por la atmósfera, lo que se espera, tarde o temprano, es un posible colapso. La crisis resultante supone inevitables calamidades sociales y naturales, de modo que no se debe seguir sobrecargando la biosfera sin antes considerar sus límites y replantear los patrones de consumo y utilización de los recursos naturales.

Dos herencias otorgadas por la sociedad moderna a las futuras generaciones comúnmente reconocidas son el cambio climático y la escasez de energía (GUPTA Y TUOHY, 2013, p. 3). No obstante, aún no se sabe cuál de ellas cobrará su precio en primer lugar, si es que ya no se manifiestan conjuntamente en los días actuales. El reconocimiento de este doble desafío consagra formalmente la búsqueda de objetivos asociados a las energías renovables para satisfacer la ascendente demanda de energía y reducir los impactos negativos tanto en el ámbito internacional como nacional. Precisamente, la Agenda 2020 de Desarrollo Sostenible de la ONU, que prevé "adoptar medidas urgentes para combatir el cambio climático y sus efectos" (ONU, 2015, objetivo 13) y el Protocolo de Kioto ${ }^{4}$, derivan en políticas y planes energéticos a nivel local.

Mientras que, por un lado, la crisis energética mundial se atribuye a la disminución sustancial de los depósitos de combustibles fósiles y la volatilidad de los precios mundiales, por otro, el uso intensivo de estos combustibles para la generación de energía resulta en altas emisiones de dióxido de carbono a la atmósfera, con efectos asociados al calentamiento global. En su informe más destacado, el Grupo Intergubernamental de Expertos sobre el Cambio Climático de las Naciones Unidas sugirió que los combustibles fósiles se deberían eliminar por completo hasta 2100 (PACHAURI et al, 2014, p. 8). El informe indica que la energía renovable

4 Basándose en los principios de la Convención Marco de las Naciones Unidas sobre el Cambio Climático, el Protocolo de Kioto compromete a los países industrializados a estabilizar las emisiones de gases de efecto invernadero, a través de la constitución de metas vinculantes de reducción de las emisiones para 37 países industrializados y la Unión Europea, reconociendo que son los principales responsables de los elevados niveles de emisiones de gases de efecto invernadero actualmente existentes en la atmósfera, y que son el resultado de quemar combustibles fósiles durante más de 150 años (ONU, 2017, par. 1-2). 
en 2014 representaba alrededor del 30\% del suministro mundial de energía, que se debería incrementar en al menos un $80 \%$ hasta 2050 si se desea alcanzar el objetivo propuesto.

Entre los principales combustibles fósiles se identifican el carbón, el gas natural y el petróleo. Este último fue el responsable de un 39,9\% del consumo global de combustibles en 2014, con una concentración del $64,5 \%$ en el sector del transporte (AIE, 2016, p. 28 y 33). Por esta razón, a principios del siglo XXI los biocarburantes se reconocieron como la alternativa más atractiva y práctica para reemplazar a los derivados del petróleo en el transporte, ya que pueden utilizar la misma red logística de distribución y no implican cambios muy radicales en las políticas de transporte y la tecnología actualmente empleada en los vehículos automotores.

Para Sánchez, López, Pérez y Rincón (2006, p. 35), los biocarburantes forman parte de la categoría general abarcada por el término biocombustible y lo definen como "aquellos biocombustibles susceptibles de ser empleados en un motor de combustión interna". Colectivamente se denominan "biocarburantes de primera generación", también conocidos como "convencionales", aquéllos producidos a partir de productos alimentarios, como el maíz, la colza, la soja, la remolacha, los cereales o la caña de azúcar, y son los únicos comercializados a gran escala a nivel internacional. Considerando que absorben el carbono de la atmósfera en la etapa agrícola de producción, tienen el potencial de compensar las emisiones de dióxido de carbono y mitigar el cambio climático.

Con base en tal escenario, mundialmente se fijaron metas muy ambiciosas relativas al desarrollo de estos combustibles alternativos y, como resultado, entre 2000-2010 su producción experimentó un incremento del 900\% (AIE, 2017). En particular, en la Unión Europea (UE), se estableció un importante objetivo de sustitución de un $10 \%$ de combustibles fósiles por fuentes renovables - esencialmente biocarburantes - en el sector de los transportes para 2020.

Para cumplirlo, se previó un aumento de la producción doméstica de biodiésel, acompañado de un incremento de la importación de etanol (AL-RIFFAI, DIMARANAN Y LABORDE, 2010, p. 11). Sin embargo, las predicciones chocaron con una amplia controversia a nivel público, político y científico acerca de los impactos negativos ambientales y sociales de los cultivos energéticos. El debate arrojó dudas sobre la sostenibilidad de la expansión de la demanda de la primera generación 
de esta energía, que aumenta la presión para intensificar la producción agrícola generando una potencial amenaza a la seguridad alimentaria ${ }^{5}$ e impactos como la degradación de los suelos, el uso insostenible del agua, la contaminación del aire, la reducción de la biodiversidad, el desplazamiento de poblaciones indígenas y rurales, además de violaciones de Derechos Humanos (KOIZUMI, 2015, p. 830).

Además, se cierne sobre esta generación de biocarburantes otra importante preocupación, relacionada con los efectos negativos asociados al cambio indirecto del uso de la tierra. Según Eurobserv'er (2015, p. 5), se ha demostrado que un aumento global del consumo de materias primas agrícolas por parte del sector energético, compensado por el cultivo en parcelas no dedicadas originalmente a la agricultura - áreas forestales, praderas naturales, turberas, etc.-, tiene el potencial de producir emisiones adicionales de gases de efecto invernadero en todo el mundo.

El reto para los Estados es equilibrar la demanda de energía y el desarrollo sostenible con la reducción de la producción de energía fósil y la expansión de la producción de biocombustibles. Se propone un estudio para analizar la evolución de las regulaciones europeas para los biocombustibles sostenibles. Esta investigación se realizó con la revisión bibliográfica de fuentes primarias y secundarias, basada en textos normativos de la Unión Europea e informes producidos por sus oficinas oficiales.

\section{EVOLUCION DE LA POLÍTICA EUROPEA PARA LOS BIOCARBURANTES}

En la UE, estas preocupaciones se tradujeron en una fuerte oposición a la producción de la primera generación de biocarburantes y el incentivo a un desarrollo cauteloso de las generaciones más avanzadas ${ }^{6}$, acarreando una desaceleración de la producción y el consumo de esta fuente de energía (BOURGUIGNON, 2015, p. 3). La Directiva 2009/28/CE entró en vigor en el auge de esta polémica, orientando el foco de la política europea de biocarburantes hacia la sostenibilidad.

5 Según la definición de la FAO (1996) "existe seguridad alimentaria cuando todas las personas tienen en todo momento acceso físico y económico a suficientes alimentos inocuos y nutritivos para satisfacer sus necesidades alimenticias y sus preferencias en cuanto a los alimentos a fin de llevar una vida activa y sana".

6 "Les biocarburants des première génération sont représentés notamment par le biodiesel et le bioéthanol." (...)"Les biocarburants dits "avancés" appartient géneéralement à la deuxième gereration. Ils sont produits a partir des matières ligno-cellulosique des plantes et des déchets, comme par exemple, huile usagée." (ANDRADE, P. Le developpement durable des biocarburants: objet d'un droit transnational. Paris: L'Harmattan, 2018. p. 23-24) 


\section{La tabla 1 presenta una visión global del escenario político normativo} en el cual se desarrolló política europea de biocarburantes.

Tabla 1 Evolución de la política europea de biocarburantes

\begin{tabular}{|c|c|c|}
\hline Fecha & Documento & Directiva/documento político \\
\hline 13/10/1998 & 98/70/CE & Directiva relativa a la calidad de la gasolina y el gasóleo \\
\hline $7 / 11 / 2001$ & $\begin{array}{l}\operatorname{COM}(2001) \\
547 \text { final }\end{array}$ & $\begin{array}{l}\text { Comunicación de la Comisión al Parlamento Europeo, al } \\
\text { Consejo, al Comité Económico y Social y al Comité de } \\
\text { las Regiones relativa a los combustibles alternativos para } \\
\text { el transporte por carretera y a un conjunto de medidas } \\
\text { para promover el uso de biocarburantes }\end{array}$ \\
\hline $3 / 03 / 2003$ & 2003/17/CE & $\begin{array}{l}\text { Directiva que modifica la Directiva } 98 / 70 / \text { CE relativa a la } \\
\text { calidad de la gasolina y el gasóleo }\end{array}$ \\
\hline $8 / 05 / 2003$ & 2003/30/CE & $\begin{array}{l}\text { Directiva relativa al fomento del uso de biocarburantes u } \\
\text { otros combustibles renovables en el transporte }\end{array}$ \\
\hline $7 / 12 / 2005$ & $\begin{array}{l}\operatorname{COM}(2005) \\
628 \text { final }\end{array}$ & Plan de acción sobre la biomasa \\
\hline $8 / 02 / 2006$ & $\begin{array}{l}\operatorname{COM}(2006) 34 \\
\text { final }\end{array}$ & Estrategia de la UE para los biocarburantes \\
\hline $\begin{array}{l}\text { Abril - } \\
\text { julio/2006 }\end{array}$ & & Consulta pública de revisión de la Directiva 2003/30/CE \\
\hline $10 / 01 / 2007$ & $\begin{array}{l}\operatorname{COM}(2006) \\
848\end{array}$ & $\begin{array}{l}\text { Programa de trabajo de la energía renovable - Las } \\
\text { energías renovables en el siglo XXI: construcción de un } \\
\text { futuro más sostenible }\end{array}$ \\
\hline $\begin{array}{l}16 / 05 / 2007 \text { al } \\
18 / 06 / 2007\end{array}$ & & $\begin{array}{l}\text { Consulta pública concerniente a los biocarburantes dentro } \\
\text { de la nueva legislación sobre la promoción de la energía } \\
\text { renovable }\end{array}$ \\
\hline $10 / 012007$ & $\begin{array}{l}\mathrm{COM}(2006) \\
845 \text { final }\end{array}$ & $\begin{array}{l}\text { Informe sobre los progresos realizados respecto de } \\
\text { la utilización de biocarburantes y otros combustibles } \\
\text { renovables en los EM de la UE }\end{array}$ \\
\hline $31 / 01 / 2007$ & $\begin{array}{l}\operatorname{COM}(2007) 18 \\
\text { final }\end{array}$ & $\begin{array}{l}\text { Propuesta de Directiva del Parlamento Europeo y del } \\
\text { Consejo por la que se modifica la Directiva 98/70/CE en } \\
\text { relación con las especificaciones de la gasolina, el diésel } \\
\text { y el gasóleo, se introduce un mecanismo para controlar } \\
\text { y reducir las emisiones de gases de efecto invernadero } \\
\text { derivadas de la utilización de combustibles de transporte } \\
\text { por carretera }\end{array}$ \\
\hline $23 / 01 / 2008$ & $\begin{array}{l}\operatorname{COM}(2008) 19 \\
\text { final }\end{array}$ & $\begin{array}{l}\text { Propuesta de Directiva del Parlamento Europeo y del } \\
\text { Consejo relativa al fomento del uso de energía procedente } \\
\text { de fuentes renovables }\end{array}$ \\
\hline $17 / 09 / 2008$ & 2009/C 77/12 & $\begin{array}{l}\text { Dictamen del Comité Económico y Social Europeo sobre } \\
\text { la "Propuesta de Directiva del Parlamento Europeo y del } \\
\text { Consejo relativa al fomento del uso de energía procedente } \\
\text { de fuentes renovable" }\end{array}$ \\
\hline $17 / 12 / 2008$ & & $\begin{array}{l}\text { Parlamento Europeo vota la Directiva relativa al fomento } \\
\text { del uso de energía procedente de fuentes renovables }\end{array}$ \\
\hline $6 / 04 / 2009$ & $52^{\circ}$ año, L140 & $\begin{array}{l}\text { Publicación de la Directiva relativa al fomento del uso } \\
\text { de energía procedente de fuentes renovables en el Diario } \\
\text { Oficial de la UE }\end{array}$ \\
\hline
\end{tabular}




\begin{tabular}{|l|c|l|}
\hline $23 / 04 / 2009$ & $2009 / 28 / \mathrm{CE}$ & $\begin{array}{l}\text { Directiva relativa al fomento del uso de energía procedente } \\
\text { de fuentes renovables }\end{array}$ \\
\hline $23 / 04 / 2009$ & $2009 / 30 / \mathrm{CE}$ & $\begin{array}{l}\text { Modifica la Directiva 98/70/CE, relativa a la calidad de } \\
\text { la gasolina y el gasóleo, introduciendo la regulación de la } \\
\text { sostenibilidad de los biocarburantes }\end{array}$ \\
\hline $9 / 09 / 2015$ & (UE) $2015 / 1513$ & $\begin{array}{l}\text { Modifica la Directiva 98/70/CE, relativa a la calidad de la } \\
\text { gasolina y el gasóleo, y la Directiva 2009/28/CE, relativa } \\
\text { al fomento del uso de energía procedente de fuentes } \\
\text { renovables }\end{array}$ \\
\hline
\end{tabular}

Fuente: GRINSVEN, 2009

La promoción de los biocarburantes en la UE tiene como punto de partida la "Comunicación de la Comisión al Parlamento Europeo, al Consejo, al Comité Económico y Social y al Comité de las Regiones relativa a los combustibles alternativos para el transporte por carretera y a un conjunto de medidas para promover el uso de biocarburantes" (COM 2001), a partir de la cual se reconoció a los biocarburantes como fuente alternativa a la utilización de los combustibles fósiles para el transporte por carretera.

Dos años después, la Directiva 2003/17/CE revisó la Directiva 98/70/ $\mathrm{CE}$, referente a la calidad de la gasolina y el gasóleo, previendo la mezcla de biocarburantes a los derivados de petróleo, establecida en un 5\% por volumen de combustible. Sin embargo, esta mezcla no era vinculante y no se determinaba un plazo de implantación. Fue en la Directiva 2003/30/ $\mathrm{CE}$, orientada a fomentar el uso de biocarburantes $\mathrm{u}$ otros combustibles renovables en el transporte, cuando por primera vez se fijaron metas para su uso en los Estados miembros, que tenían como fundamento tanto en la seguridad energética como la reducción de gases de efecto invernadero.

A tal fin, como objetivo general se determinó "el cumplimiento de los compromisos asumidos en materia de cambio climático, la seguridad de abastecimiento en condiciones ecológicamente racionales y la promoción de las fuentes de energía renovables" (artículo 1, Directiva 2003/30/CE). Se constituyeron valores de referencia no vinculantes a los porcentuales de sustitución de los derivados fósiles en la UE, entre el 2 y el 5,75\%, calculados sobre la base del contenido energético de todo el gasóleo y la gasolina destinados al transporte, con fechas límites para el cumplimiento de cada porcentual.

Por añadidura, se facultó a los Estados miembros la elección entre la mezcla o la utilización integral de los biocarburantes, por lo que las mezclas superiores al 5\% deberían ser indicadas en el producto a través de 
un sello. No obstante, pese a todas las iniciativas nacionales establecidas, el gobierno holandés fue el único en lograr implementar estos objetivos en su legislación nacional en el año 2005 (GRINSVEN, 2009, p. 34). Esto se debe a que en la mayoría de los países de la UE fue necesario crear la cadena de producción y suministro de biocarburantes a partir de cero.

Con base en este resultado, la Comisión desarrolló la "Estrategia de la Unión Europea para los Biocarburantes" (COM 2006), que se apoyaba en el anterior "Plan de Acción sobre la Biomasa" (COM 2005). Entre los elementos fundamentales de este plan se destacaba la necesidad de reducir la demanda de energía, incrementar la confianza en las fuentes de energía renovables, diversificar las fuentes de energía y promover la cooperación internacional.

En este período, como se aprecia en el gráfico 1, la UE se mostraba autosuficiente en la producción y el consumo de biocarburantes. Las políticas nacionales se centraban en la producción local y su objetivo era eliminar las barreras técnicas, económicas y sociales para la expansión del mercado de estos combustibles. Según Di Lucia (2013, p. 83),

mediante la vinculación de políticas en favor de los biocarburantes para el desarrollo rural y la autosuficiencia energética, los gobiernos atrajeron el apoyo político de las organizaciones de agricultores y grupos de presión industriales, generando un ascenso de la producción de biocarburantes en la UE.

Gráfico 1 Consumo y producción de biocarburantes en la UE (2005-2014).

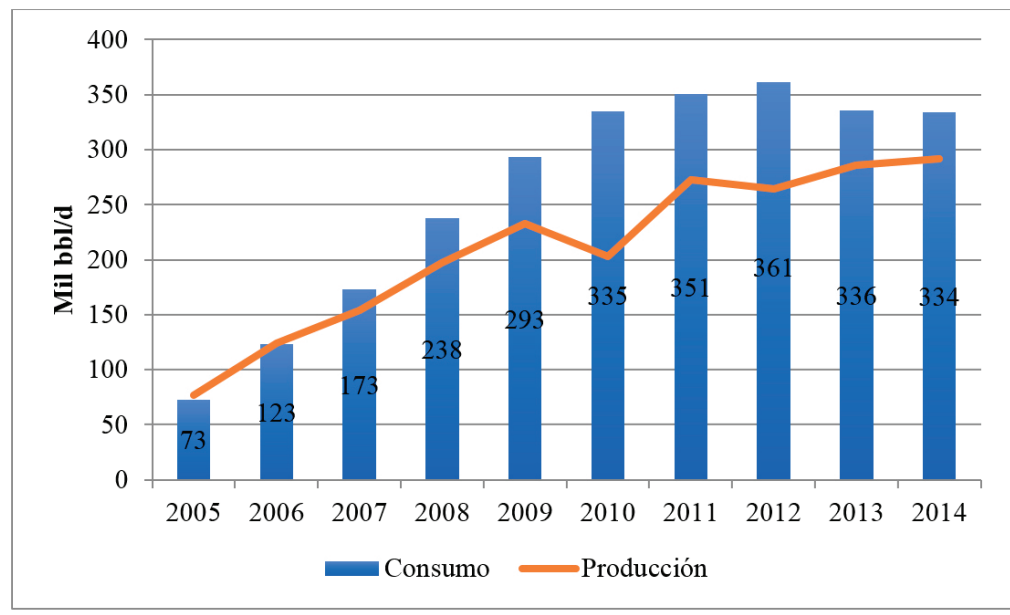

Fuente: EIA (2017). 
Esta primera etapa de desarrollo de la política europea, que se puede situar entre 2005-2007, tuvo como protagonistas los gobiernos nacionales, la industria de biocarburantes y automotora, los agricultores y las ONG ambientalistas. Pese a que algunas ONG empezaran a cuestionar los beneficios de destinar cultivos agrícolas al uso energético, el conjunto de estos actores manifestaba estar de acuerdo con la importancia de expandir la producción de esta fuente alternativa en el sector del transporte (LONDO, DEURWAARDER Y VAN THUIJL, 2006, p. 3).

En el referido escenario, la escala de la producción y el consumo era local, es decir, se concentraba en el territorio europeo. La UE se preparaba paradestinarmayores cantidades de biocarburantes al transporte, impulsando la sustitución del petróleo y el desarrollo rural, pero la preocupación por la sostenibilidad todavía era muy incipiente. A este respecto, la revisión de la Directiva 2003/30/CE iniciada en 2007 apuntaba a un nuevo período en la política europea de esta fuente de energía renovable.

Entre 2007-2009, con la industria consolidada y la producción en considerable ascensión, una gama más amplia de partes interesadas pasó a conformar el panorama de esta fuente de energía en la UE, que incluya tanto a los actores directamente involucrados en la cadena de producción y suministro como a los afectados de manera indirecta por la misma (DI LUCIA, 2013, p. 85). Destaca que en esta fase la mayoría de los grupos de interés disponía de posiciones bien definidas, pero muy divergentes, sobre los biocarburantes, en particular en relación con los beneficios y riesgos de la producción intensiva (BOURGUIGNON, 2015, p. 4).

Además, los actores se distribuían por todo el mundo, incluso en zonas geográficamente desconectadas de la producción física del combustible renovable. Por esta razón, se empezó a otorgar especial relevancia a los impactos asociados a la expansión de la producción, lo que resultó en el establecimiento de una política europea de control y mitigación de los efectos negativos de la producción y consumo de los biocarburantes (DI LUCIA, 2013, p. 85), consolidada en la Directiva 2009/28/CE.

A tal fin, la Directiva de 2009, que forma parte del "Paquete de medidas sobre clima y energía hasta 2020" (COM 2008a), se presentó como una iniciativa pionera al desarrollar un meta-estándar de criterios de sostenibilidad e introducir un objetivo del uso de un $10 \%$ de energías renovables - esencialmente biocarburantes - para sustituir al petróleo en el sector de los transportes antes de 2020. De modo complementario a la predecesora Directiva 2003/30/CE, el principal avance de este nuevo 
instrumento normativo consistió en dibujar un vínculo explícito entre el consumo de biocarburantes y la producción sostenible (LYDGATE, 2013, p. 159$){ }^{7}$

\section{LA DIRECTIVA DE ENERGÍAS RENOVABLES DE LA UNIÓN EUROPEA}

La Directiva 2009/28/CE, del Parlamento Europeo y del Consejo, entró en vigor el 25 de junio de 2009 con la transposición a la legislación nacional prevista para el 5 de diciembre de 2010. Constituye la Directiva guía de la "Política de Energías Renovables de la Unión Europea hasta el 2020", y en el ámbito mundial es la primera que presenta criterios de sostenibilidad vinculantes para la producción de biocarburantes. Adoptada a iniciativa de la Dirección General de Energía y Transportes de la UE, su objetivo principal es el desarrollo de las energías procedentes de fuentes renovables, vinculado a una mejora en la eficiencia energética para reducir las emisiones de GEI.

De esa manera prevé, por una parte, alcanzar una cuota del $20 \%$ de energía procedente de fuentes renovables en el consumo total de energía de la UE y, por otra, una cuota vinculante mínima del $10 \%$ para cada Estado miembro respecto del porcentaje de biocarburantes consumidos en el transporte hasta el 2020 (Artículo 3.4). Este incentivo específico del uso de la energía procedente de fuentes renovables en el transporte se orienta "a reducir la dependencia de la UE de las importaciones de petróleo en el sector, ámbito en el que el problema de la seguridad del abastecimiento de energía es especialmente agudo, e influir en el mercado de los combustibles para el transporte" (Considerando 2).

La Directiva fija el mismo objetivo para todos los Estados miembros, con el fin de garantizar la coherencia de las especificaciones aplicables a los biocarburantes y su disponibilidad. Además, señala la necesidad de fomentar la eficiencia energética en el sector del transporte, dada "la probabilidad de que el porcentual obligatorio de la energía procedente

\footnotetext{
7 Al mismo tiempo, una enmienda a la Directiva 98/70/CE - la Directiva sobre la Calidad de los Combustibles - a través de la Directiva 2009/30/CE introdujo el objetivo obligatorio de reducir en un 6\% la intensidad de los GEI de los combustibles utilizados en el transporte por carretera. Esta Directiva modificó una serie de elementos de las especificaciones del gasóleo y la gasolina, así como introdujo en el artículo 7bis requisitos a los proveedores de combustible para reducir la intensidad de la emisión de GEI en el sector del transporte por carretera. También estableció en el artículo 7ter los mismos criterios de sostenibilidad para los biocarburantes previstos en la Directiva, que se deberían cumplir para contribuir al objetivo de reducir las emisiones de GEI durante el ciclo de vida de los combustibles hasta el $10 \%$ por unidad de energía del combustible o por energía suministrada.
} 
de fuentes renovables sea cada vez más difícil de alcanzar de manera sostenible si sigue aumentando la demanda global de energía para el transporte" (Considerando 1, Directiva (UE) 2015/1513).

Respecto del comercio internacional, el Considerando 16 establece que:

[...] si bien para la Comunidad sería técnicamente posible cumplir el objetivo en materia de uso de energía procedente de fuentes renovables en el transporte solamente a partir de su producción interna, es a la vez probable y deseable que alcance este objetivo combinando la producción interna y las importaciones.

Por ello, la Directiva determina que la Comisión supervise el suministro del mercado europeo de biocarburantes, y que proponga medidas pertinentes para lograr un equilibrio entre la producción nacional y las importaciones, teniendo en cuenta "el desarrollo de negociaciones comerciales multilaterales y bilaterales, así como consideraciones medioambientales, sociales, económicas y de seguridad en el suministro de energía, entre otras." La Directiva dispone explícitamente respecto algunos problemas ambientales relacionados con los biocombustibles, identificándolos como el impacto sobre el cambio climático y la conservación de la biodiversidad. Sin embargo, no menciona otros como la erosión del suelo, la contaminación del agua, o el uso de los defensivos agrícolas (ANDRADE, 2018, p. 158).

En tal contexto, se establece en el artículo 17, apartados 2-6, un meta-estándar de criterios vinculantes de sostenibilidad relacionados con la producción de esta fuente de energía, cuya observancia es vinculante tanto para evaluar el cumplimiento de los objetivos nacionales como las obligaciones de utilizar energías renovables y poder optar por una ayuda financiera al consumo. La Directiva alienta el consumo de biocombustibles que contribuyen a la reducción de las emisiones de gases de efecto invernadero al garantizar que se cumplan los objetivos para reducir el efecto de la producción de tales contaminantes en el cambio climático. La tabla 2 reseña los criterios medioambientales de sostenibilidad ${ }^{8}$ abarcados $^{2}$ por la Directiva.

8 Es importante señalar que la Directiva 2009/28/CE también prevé criterios sociales de sostenibilidad en su artículo 17.7 (tabla 4), pero para estos establece un marco débil de verificación, con la presentación de informes cada dos años por la Comisión al Parlamento Europeo y al Consejo en relación con los terceros países y los Estados miembros que constituyan una fuente importante de materias primas o de biocarburantes consumidos en la UE. A diferencia de los criterios medioambientales, los criterios sociales no tienen fuerza vinculante, son imprecisos y carecen de mecanismos de verificación (Portocarrero y Ribeiro, p. 4, 2012). 
Tabla 2 Criterios de sostenibilidad medioambiental

\begin{tabular}{|c|c|}
\hline $\begin{array}{l}\text { Reducción de las } \\
\text { emisiones de GEI } \\
\text { (artículo 17.2) }\end{array}$ & $\begin{array}{l}\text { La reducción de las emisiones de GEI Directiva del uso de } \\
\text { biocarburantes será de un } 60 \% \text { como mínimo en el caso de los } \\
\text { biocarburantes producidos en instalaciones que empiecen a estar } \\
\text { operativas después del } 5 \text { de octubre de } 2015 \text {. Se considerará } \\
\text { que una instalación está operativa cuando haya tenido lugar la } \\
\text { producción física de biocarburantes o biolíquidos. En el caso de } \\
\text { las instalaciones que estén operativas el } 5 \text { de octubre de } 2015 \text { o } \\
\text { antes de esa fecha, la reducción de las emisiones de GEI será de } \\
\text { un } 35 \% \text { como mínimo hasta el } 31 \text { de diciembre de } 2017 \text {, y del } \\
50 \% \text { como mínimo a partir del } 1 \text { de enero de } 2018 \text {. }\end{array}$ \\
\hline $\begin{array}{l}\text { Tierras de elevado } \\
\text { valor en cuanto } \\
\text { a biodiversidad } \\
\text { (artículo 17.3) }\end{array}$ & $\begin{array}{l}\text { Los biocarburantes no se producirán a partir de materias } \\
\text { primas procedentes de tierras de elevado valor en cuanto a } \\
\text { biodiversidad, es decir tierras que a partir de enero de } 2008 \\
\text { pertenecían a una de las siguientes categorías, con independencia } \\
\text { de que sigan encontrándose en la misma situación: a) bosques } \\
\text { primarios y otras superficies boscosas de especies nativas, } \\
\text { cuando no hay signos visibles claros de actividad humana y los } \\
\text { procesos ecológicos no están perturbados significativamente; b) } \\
\text { zonas designadas: i) por ley o por las autoridades competentes } \\
\text { pertinentes con fines de protección de la naturaleza, o ii) para la } \\
\text { protección de las especies o los ecosistemas raros, amenazados o } \\
\text { en peligro, reconocidos por acuerdos internacionales o incluidos } \\
\text { en listas elaboradas por organizaciones intergubernamentales o } \\
\text { la Unión Internacional para la Conservación de la Naturaleza, } \\
\text { a condición de que dichas zonas hayan sido reconocidas de } \\
\text { conformidad con el artículo } 18.4 \text {, párrafo segundo, a menos } \\
\text { que se demuestre que la producción de tales materias primas no } \\
\text { ha interferido con dichos fines de protección de la naturaleza; c) } \\
\text { prados y pastizales con una rica biodiversidad: i) que seguirían } \\
\text { siéndolo a falta de intervención humana y que conservan la } \\
\text { composición en especies naturales y las características y procesos } \\
\text { ecológicos, o ii) que dejarían de serlo a falta de intervención } \\
\text { humana, que son ricos en especies y no están degradados, salvo } \\
\text { que se demuestre que la explotación de las materias primas es } \\
\text { necesaria para preservar su condición de prados y pastizales. }\end{array}$ \\
\hline $\begin{array}{l}\text { Tierras con elevadas } \\
\text { reservas de carbono } \\
\text { (artículo 17.4) }\end{array}$ & $\begin{array}{l}\text { Los biocarburantes no se fabricarán a partir de materias primas } \\
\text { procedentes de tierras con elevadas reservas de carbón, es decir } \\
\text { tierras que en enero de } 2008 \text { pertenecían a una de las siguientes } \\
\text { categorías pero que ya no se encuentran en dicha situación: a) } \\
\text { humedales, es decir, tierras cubiertas de agua o saturadas por } \\
\text { agua permanentemente o durante una parte importante del año; } \\
\text { b) zonas arboladas continuas, es decir tierras con una extensión } \\
\text { superior a una hectárea, con árboles de una altura superior a } \\
\text { cinco metros y una cubierta de copas superior al } 30 \% \text {, o con } \\
\text { árboles que pueden alcanzar dichos límites in situ; c) tierras con } \\
\text { una extensión superior a una hectárea, con árboles de una altura } \\
\text { superior a cinco metros y una cubierta de copas de entre el } 10 \% \\
\text { y el } 30 \% \text { o con árboles que pueden alcanzar dichos límites in } \\
\text { situ, salvo si se aportan pruebas de que las reservas de carbono } \\
\text { de la zona en cuestión antes y después de la conversión son tales } \\
\text { que, cuando se aplica la metodología contemplada en el anexo V, } \\
\text { parte C, se cumplen las condiciones establecidas en el apartado } 2 \\
\text { del artículo } 17 \text { de la Directiva. }\end{array}$ \\
\hline
\end{tabular}




\begin{tabular}{|c|c|}
\hline $\begin{array}{l}\text { Tierras turberas } \\
\text { (artículo 17.5) }\end{array}$ & $\begin{array}{l}\text { Los biocarburantes no provendrán de materias primas extraídas } \\
\text { de tierras que, a enero de } 2008 \text {, fueran turberas, a no ser que } \\
\text { se aporten pruebas de que el cultivo y la recolección de esta } \\
\text { materia prima no implican el drenaje de suelos no drenados con } \\
\text { anterioridad. }\end{array}$ \\
\hline $\begin{array}{l}\text { Reglamento } \\
\text { (CE) } \mathrm{n}^{\circ} 73 / 2009 \\
\text { (artículo 17.6) }\end{array}$ & $\begin{array}{l}\text { Las materias primas agrícolas cultivadas en la UE y utilizadas } \\
\text { para la producción de biocarburantes se obtendrán de } \\
\text { conformidad con los requisitos y normas previstos en las } \\
\text { disposiciones a que se refiere el título "Medio ambiente" en la } \\
\text { parte A y en el punto } 9 \text { del anexo II del Reglamento (CE) no } \\
73 / 2009 \text { del Consejo, de } 19 \text { de enero de } 2009 \text {, por el que se } \\
\text { establecen disposiciones comunes aplicables a los regímenes de } \\
\text { ayuda directa a los agricultores en el marco de la política agrícola } \\
\text { común y se instauran determinados regímenes de ayuda a los } \\
\text { agricultores, y de conformidad con los requisitos mínimos de las } \\
\text { buenas condiciones agrarias y medioambientales definidos con } \\
\text { arreglo al artículo 6, apartado } 1 \text {, de dicho Reglamento. }\end{array}$ \\
\hline
\end{tabular}

Fuente: Directiva 2009/28/CE.

Respecto de los mecanismos disponibles para verificar estas exigencias de sostenibilidad, los productores de los terceros países disponen de tres herramientas: acuerdos bilaterales o multilaterales; sistemas nacionales establecidos por los Estados miembros; o regímenes voluntarios de certificación reconocidos por la Comisión Europea. Entre estas opciones, la certificación es el mecanismo de uso más extendido, ya que confiere a los productores la seguridad de que sus cultivos cumplirán con los requisitos de todos los Estados miembros, independientemente del origen de la materia prima (COM 2015, p. 15).

Asimismo, el artículo 4 dispone que los Estados miembros deberán "adoptar un plan de acción nacional en materia de energías renovables, que determinará sus objetivos nacionales en relación con las cuotas de energía procedentes de fuentes renovables consumidas en el transporte en 2020." A tal fin, deben tener en cuenta las medidas adoptadas para alcanzar dichos objetivos globales nacionales, que comprenden

la cooperación entre autoridades locales, regionales y nacionales, las transferencias estadísticas o los proyectos conjuntos programados, las estrategias nacionales destinadas a desarrollar los recursos de biomasa existentes y a movilizar nuevos recursos de biomasa para usos diferentes, así como las medidas que deberán adoptarse para cumplir los requisitos de los artículos 13 a 19. (Directiva 2009/28/CE)

La Comisión Europea ofrece un modelo para los planes de acción nacionales que recoge los requisitos mínimos establecidos en la Directiva. Estos planes se difunden cada dos años en la plataforma de transparencia 
de la UE, acompañados de un informe relativo al progreso de la energía renovable en los Estados miembros. ${ }^{9}$ El último informe se presentó en febrero de 2017 (COM 2017).

De acuerdo con el referido informe, 25 de los 28 Estados miembros habían incorporado los criterios de sostenibilidad de la Directiva a su legislación nacional en 2014. Sin embargo, de estos países tan solo 19 lograron sustituir hasta un $5 \%$ de combustibles fósiles por biocarburantes en el transporte. En particular, Austria, Suecia y Finlandia figuran como los únicos Estados miembros a alcanzar la meta establecida, con una cuota del $10,9 \%$, el $21,1 \%$ y el $22 \%$, respectivamente. De este total, el biodiésel destaca como la energía renovable más utilizada en el transporte, con 12.7 Mtep, seguido del etanol, con 3.5 Mtep (EUROSTAT, 2017).

\section{FACTORES CONDICIONANTES DE LA INDUSTRIA DE BIOCARBURANTES EN LA UNIÓN EUROPEA}

Cuando se adoptó la Directiva 2009/28/CE, el Parlamento Europeo y el Consejo solicitaron a la Comisión Europea que examinara el impacto indirecto de las emisiones de gases de efecto invernadero derivadas de la expansión de los cultivos agrícolas para la producción de los biocarburantes de primera generación y que, en su caso, presentara una propuesta legislativa. Este tema, sumamente controvertido, se sometió a un largo debate normativo culminando en la adopción de la Directiva (UE) 2015/1513, que cambiaría la trayectoria de la política europea de esta fuente renovable.

El proyecto de esta nueva Directiva, destinada a limitar la participación de la primera generación de biocarburantes en el consumo energético europeo a un 5\%, se presentó en octubre de 2012 por la Comisión Europea al Parlamento Europeo. El nuevo umbral establecido implicaba detener la producción de la primera generación de estos combustibles en Europa (EUROBSERV'ER, 2015, p. 5). Por esta razón, la propuesta despertó la insatisfacción de la industria de biocarburantes, al desestimar las altas inversiones ya realizadas para la implementación de los objetivos de las Directivas antecedentes.

Como respuesta a esta insatisfacción, en 2014 el Consejo Europeo de Ministros de Energía estableció un acuerdo político con el sector, cuyas

9 Los planes de acción y los informes de la CE están disponibles en http://ec.europa.eu/energy/en/ topics/renewable-energy/progress-reports, en 13 de junio de 2017. 
características principales se plasmaron en las modificaciones aportadas por la Directiva (UE) 2015/1513 a la Directiva 2009/28/CE (PRESSE 138, 2014). El principal efecto de esas modificaciones consistió en limitar la participación de los biocarburantes de primera generación a un 7\% en los Estados miembros. Se mantuvo el objetivo global del 10\% de energías renovables en el transporte, por lo que el 3\% restante se debería obtener a través de la movilidad eléctrica o de los biocarburantes avanzados, cuya contribución a la consecución del objetivo global se consideraría el doble de su contenido en energía.

Sin embargo, la inseguridad generada por este largo proceso de revisión normativa afectó de modo negativo al mercado de los biocarburantes en la UE. Según se aprecia en la Tabla 3, aunque entre 2005-2014 se observe un aumento global en la utilización de las energías renovables en los sistemas urbanos de calefacción, refrigeración y electricidad, en el sector del transporte los avances hacia la consecución del objetivo de la Directiva 2009/28/CE han sido lentos. El último informe relativo al progreso de la energía renovable, presentado por la Comisión en 2017 (COM 2017, p. 8), revela que en 2014 se alcanzó una cuota del 6,5\% respecto del objetivo de sustituir un $10 \%$ de los combustibles fósiles por biocarburantes.

Tabla 3 Porcentaje de participación de la energía procedente de fuentes renovables por sector (2005-2014)

\begin{tabular}{|l|l|l|l|l|l|l|l|l|l|c|}
\hline Sector & $\mathbf{2 0 0 5}$ & $\mathbf{2 0 0 6}$ & $\mathbf{2 0 0 7}$ & $\mathbf{2 0 0 8}$ & $\mathbf{2 0 0 9}$ & $\mathbf{2 0 1 0}$ & $\mathbf{2 0 1 1}$ & $\mathbf{2 0 1 2}$ & $\mathbf{2 0 1 3}$ & $\mathbf{2 0 1 4}$ \\
\hline Transporte & 1,8 & 2,5 & 3,1 & 3,9 & 4,6 & 5,2 & 4,0 & 5,6 & 5,9 & 6,5 \\
\hline Electricidad & 14,8 & 15,4 & 16,1 & 17,0 & 19,0 & 19,7 & 21,7 & 23,5 & 25,4 & 27,5 \\
\hline $\begin{array}{l}\text { Calefacción y } \\
\text { refrigeración }\end{array}$ & 10,9 & 11,4 & 12,8 & 13,1 & 14,7 & 14,9 & 15,6 & 16,4 & 16,9 & 18,1 \\
\hline
\end{tabular}

Fuente: Eurostat, 2017.

La Comisión (COM 2015, p. 3) asocia expresamente estos resultados a la incertidumbre provocada por los retrasos en la finalización de la estrategia para limitar los riesgos de cambio indirecto del uso de la tierra y a los progresos insuficientes en la implantación de biocarburantes alternativos de segunda generación. En este contexto, la Directiva (UE) 2015/1513 convergió en una reorientación de la estrategia de las políticas europeas de transporte hacia las generaciones más avanzadas de esta fuente alternativa de energía, así como en la electrificación del parque de vehículos ligeros (COM 2014, p. 9). 
Otro punto de inflexión en el mercado de los biocarburantes en la UE se produjo en 2011, año en que el consumo de esta fuente de energía se asoció a la implementación de los criterios vinculantes de sostenibilidad de la Directiva 2009/28/CE. Los datos de Eurobserv'er (2013; 2015) apuntan a que la participación de los combustibles certificados fue ascendente entre 2011-2014, pasando de 11,7Mtep, en 2012, a 12,5Mtep en 2014, de modo que llegaron a representar el $92 \%$ de los biocarburantes consumidos en la UE.

Sin embargo, entre 2009-2014 el crecimiento promedio del consumo de biocarburantes empezó a reducirse de modo importante. En dicho periodo, este fue de tan sólo un 3\%, respecto del $48 \%$ experimentado en 2003-2008 (EUROSTAT, 2017). Eurobserv'er indica como principal razón de esta desaceleración que, con la adopción de la Directiva 2009/28/CE, la prioridad de los Estados miembros, más que el aumento del consumo, pasó a asociarse a la sostenibilidad.

La Tabla 4 señala que la producción de biocarburantes en la UE acompañó la misma tendencia del consumo. Esta experimentó un importante aumento entre 2005-2009, con un promedio del 25\%. Sin embargo, en el período siguiente descendió gradualmente para sufrir una caída histórica del 10,7\% en 2011, que coincide con la transposición de la Directiva 2009/28/CE a los Estados miembros.

Tabla 3 Panorama de los Biocarburantes en la UE en Mtep (2005-2014)

\begin{tabular}{|l|l|l|l|l|l|l|l|l|l|l|l|}
\hline \multicolumn{2}{|c}{} & $\mathbf{2 0 0 5}$ & $\mathbf{2 0 0 6}$ & $\mathbf{2 0 0 7}$ & $\mathbf{2 0 0 8}$ & $\mathbf{2 0 0 9}$ & $\mathbf{2 0 1 0}$ & $\mathbf{2 0 1 1}$ & $\mathbf{2 0 1 2}$ & $\mathbf{2 0 1 3}$ & $\mathbf{2 0 1 4}$ \\
\hline \multirow{4}{*}{ Consumo } & Biodiésel & 2.529 & 4.046 & 6.030 & 7.969 & 9.562 & 10.546 & 10.923 & 11.869 & 10.625 & 11.721 \\
\cline { 2 - 11 } & Etanol & 607 & 894 & 1.166 & 1.795 & 2.267 & 2.790 & 2.898 & 2.867 & 2.686 & 2.652 \\
\cline { 2 - 11 } & Total & $\mathbf{3 . 1 3 6}$ & $\mathbf{4 . 9 4 0}$ & $\mathbf{7 . 1 9 6}$ & $\mathbf{9 . 7 6 4}$ & $\mathbf{1 1 . 8 2 9}$ & $\mathbf{1 3 . 3 3 6}$ & $\mathbf{1 3 . 8 2 1}$ & $\mathbf{1 4 . 7 3 6}$ & $\mathbf{1 3 . 3 1 1}$ & $\mathbf{1 4 . 3 7 3}$ \\
\hline \multirow{5}{*}{ Producción } & Biodiésel & 2.499 & 3.674 & 5.199 & 6.401 & 7.876 & 8.931 & 8.504 & 9.111 & 9.787 & 11.361 \\
\cline { 2 - 12 } & Etanol & 480 & 741 & 1.036 & 1.492 & 1.688 & 1.972 & 1.763 & 2.046 & 2.564 & 2.274 \\
\cline { 2 - 12 } & Total & $\mathbf{3 . 3 7 3}$ & $\mathbf{5 . 4 8 6}$ & $\mathbf{7 . 3 6 2}$ & $\mathbf{8 . 7 1 6}$ & $\mathbf{1 0 . 5 1 0}$ & $\mathbf{1 1 . 7 2 5}$ & $\mathbf{1 0 . 5 9 4}$ & $\mathbf{1 1 . 4 8 2}$ & $\mathbf{1 2 . 7 4 3}$ & $\mathbf{1 3 . 9 8 2}$ \\
\hline \multirow{5}{*}{ Importación } & Biodiésel & 425 & 1.230 & 1.863 & 2.927 & 3.093 & 4.191 & 5.412 & 5.783 & 5.041 & 5.968 \\
\cline { 2 - 12 } & Etanol & 128 & 179 & 347 & 699 & 1.056 & 1.387 & 1.629 & 1.418 & 1.096 & 1.292 \\
\cline { 2 - 12 } & Total & $\mathbf{8 2 8}$ & $\mathbf{1 . 7 2 7}$ & $\mathbf{2 . 2 4 8}$ & $\mathbf{3 . 7 3 2}$ & $\mathbf{4 . 4 0 2}$ & $\mathbf{6 . 1 5 2}$ & $\mathbf{7 . 5 5 7}$ & $\mathbf{7 . 7 6 0}$ & $\mathbf{6 . 7 7 0}$ & $\mathbf{8 . 0 1 6}$ \\
\hline & Biodiésel & 383 & 861 & 925 & 1.296 & 1.430 & 2.599 & 2.890 & 3.049 & 4.004 & 5.507 \\
\cline { 2 - 11 }$y$ & Etanol & 28 & 30 & 144 & 360 & 488 & 556 & 498 & 639 & 956 & 936 \\
\cline { 2 - 11 } & Total & $\mathbf{4 1 2}$ & $\mathbf{8 9 2}$ & $\mathbf{1 . 0 6 9}$ & $\mathbf{1 . 6 5 5}$ & $\mathbf{1 . 9 1 8}$ & $\mathbf{3 . 1 5 5}$ & $\mathbf{3 . 3 9 0}$ & $\mathbf{3 . 6 8 7}$ & $\mathbf{4 . 9 5 9}$ & $\mathbf{6 . 4 4 3}$ \\
\hline
\end{tabular}

Fuente: Eurostat (2017). 
Respecto de la importación y exportación, es interesante señalar que los datos de Eurostat no confirman los pronósticos realizados cuando la Directiva 2009/28/CE entró en vigor, que predecían una mayor importación de etanol a la UE. Para cumplir con el objetivo de esta Directiva, se estimaba que el aumento previsto de la producción de biodiésel sería predominantemente doméstico, mientras que el etanol se importaría de terceros países (AL-RIFFAI, DIMARANAN Y LABORDE, 2010, p. 11).

Sin embargo, mientras entre 2005-2010 se observa una expresiva importación de biocarburantes por la UE, a partir de 2010 el bloque de 28 Estados miembros pasa a convertirse en exportador de esta fuente de energía, principalmente biodiésel. Asimismo, se aprecia que el consumo fue superior a la producción entre 2005-2010, pero se empezó a perfilar una tendencia de acercamiento entre estos indicadores en el período final del análisis, 2010-2014, que apunta a la consecución de una autonomía de en la industria de biocarburantes.

\section{CONCLUSIÓN}

A principios de 2014 la Comisión publicó una propuesta con el fin de establecer un marco estratégico en materia de clima y energía para el período 2020-2030, que abrió las negociaciones sobre la aplicación del próximo paquete vinculado a la energía y el cambio climático. En esta oportunidad, se sugirió un objetivo general para las energías renovables de un $27 \%$, que podría variar en cada Estado miembro según la política energética y los intereses nacionales. Sin embargo, la Comisión optó por no establecer un objetivo específico para el transporte.

Esta decisión ha sido objeto de fuertes críticas por parte de los representantes de la industria de los biocarburantes, ya que la falta de perspectiva incide principalmente sobre el desarrollo de las nuevas generaciones. Como resultado, la nueva Directiva de Energías Renovables cuya propuesta se presentó en febrero de 2017 por la Comisión Europea, plantea dos objetivos para el sector del transporte: garantizar la implantación rentable de la electricidad de fuentes renovables y su integración en el mercado; y desarrollar el potencial de descarbonización de los biocarburantes avanzados, aclarando el papel de los biocarburantes producidos a partir de cultivos alimentarios después de 2020.

Para llegar a estos objetivos, la Comisión realizó una consulta pública en línea sobre la sostenibilidad, que tuvo lugar entre el 10 de febrero y 
el 10 de mayo de 2016. Esta consulta se completó con una conferencia telemática de las partes interesadas, celebrada el 12 de mayo de 2016. Las contestaciones pusieron de relieve que los principales obstáculos al incremento de las energías renovables en el sector de los transportes incluyen la falta de un marco político estable para la etapa posterior a 2020; el largo debate sobre la sostenibilidad de los biocarburantes; y el elevado precio de los vehículos eléctricos.

Además, la gran mayoría de los encuestados señaló que una medida eficaz para promover los combustibles renovables sería mantener una obligación específica de incorporar su consumo. En este caso, diversas autoridades y empresas del sector público, productores de biocarburantes y empresas forestales manifestaron en contra de la imposición de nuevos requisitos de sostenibilidad para los biocarburantes, en vista del riesgo de un exceso de carga administrativa.

En esta línea, los interesados solicitaron coherencia en la imposición de criterios sociales y medioambientales a las fuentes de energía renovable, lo que supone que las exigencias aplicadas a los biocarburantes avanzados deberían ser las mismas establecidas al biogás utilizado para generar calor y electricidad, por ejemplo.

Entre las opciones para incrementar las energías renovables en el sector del transporte previstas en la propuesta, la mejor valorada fue la obligación de incorporar combustibles de transporte renovables avanzados con una reducción de los biocarburantes. Esta alternativa sirve de soporte para permitir un retorno gradual de las inversiones ya realizadas en los Estados miembros en relación con la primera generación, además de ofrecer una mayor seguridad a las futuras inversiones y a la innovación a gran escala en el sector.

Por tanto, el principal punto de acción de la nueva Directiva se orienta a la adopción de medidas que propicien un mayor despliegue de los combustibles avanzados. Es decir, el enfoque central ya no está en los biocarburantes de primera generación, sino en promover las siguientes generaciones y acelerar la electrificación de los vehículos de carretera.

Es posible identificar un avance en las regulaciones europeas para el uso de biocombustibles sostenibles. Si al principio la legislación europea se basaba en la sustitución de combustibles fósiles por biocombustibles sin promocionar el desarrollo sostenible, en una segunda fasis este requisito ya no es indirecto, implícito y se establece explícitamente en la Directiva 2009/28/CE, además de las posteriores, que establecieron límites 
para el uso de biocombustibles de primera generación y alentaron a los biocombustibles de segunda generación o avanzados. Estos avances están destinados a promocionar el desarrollo sostenible establecido en el Tratado Fundamental de la Unión Europea.

\section{BIBLIOGRAFÍA}

AIE. Key world energy statistics. Informe de la Agencia Internacional de Energía. 2016.

AL-RIFFAI, P.; DIMARANAN, B.; LABORDE, D. Global trade and environmental impact study of the EU biofuels mandate. Vol. 125. Washington, DC: IFPRI, 2010.

ANDRADE, P. Le developpement durable des biocarburants: objet d'un droit transnational. Paris: L'Harmattan, 2018.

BOURGUIGNON, Didier. EU Biofuels Policy. Dealing with Indirect Land Use Change. Informe del European Parliament Research Service, EPRS Briefing, 2015. Disponible en:http://www.europarl.europa.eu/thinktank/ en/document.html?reference=EPRS_BRI\%282015\%29545726. Acceso en: 9 abr. 2019

COMMISSION OF THE EUROPEAN COMMUNITIES. COM(2001) 547 final. Communication from the Commission to the European Parliament, the Council, the Economic and Social Committee and the Committee of the Regions on alternative fuels for road transportation and on a set of measures to promote the use of biofuels. Bruselas, 7.11.2001.

COMMISSION OF THE EUROPEAN COMMUNITIES. COM (2005) 628 final. Comunicación de la Comisión. Plan de acción sobre la biomasa. Bruselas, 07.12.2005.

COMMISSION OF THE EUROPEAN COMMUNITIES. COM (2006) 34 final. Communication from the Commission. An EU Strategy for Biofuels. Bruselas, 8.2.2006.

COMMISSION OF THE EUROPEAN COMMUNITIES. COM (2006) 845 final. Comisión de las Comunidades Europeas. Informe sobre los progresos realizados respecto de la utilización de biocarburantes y otros combustibles renovables en los Estados miembros de la Unión Europea. Bruselas, 10.1.2007. 
COMMISSION OF THE EUROPEAN COMMUNITIES. COM (2006) 848 final. Comisión de las Comunidades Europeas. Comunicación de la Comisión al Consejo y al Parlamento Europeo. Programa de trabajo de la energía renovable. Las energías renovables en el siglo XXI: construcción de un futuro más sostenible. Bruselas, 10.1.2007.

COMMISSION OF THE EUROPEAN COMMUNITIES. COM (2007) 18 final. Propuesta de Directiva del Parlamento Europeo y del Consejo por la que se modifica la Directiva 98/70/CE en relación con las especificaciones de la gasolina, el diésel y el gasóleo, se introduce un mecanismo para controlar y reducir las emisiones de gases de efecto invernadero derivadas de la utilización de combustibles de transporte por carretera. Bruselas, 31.1.2007.

COMMISSION OF THE EUROPEAN COMMUNITIES. COM (2008) 19 final. Comisión de las Comunidades Europeas. Propuesta de Directiva del Parlamento Europeo y del Consejo relativa al fomento del uso de energía procedente de fuentes renovables. Bruselas, 23.1.2008.

COMMISSION OF THE EUROPEAN COMMUNITIES. COM (2008a) 30 final. Comunicación de la Comisión al Parlamento Europeo, al Consejo, al Comité Económico y Social Europeo y al Comité de las Regiones. Dos veces 20 para el 2020. El cambio climático, una oportunidad para Europa. Bruselas, 23.1.2008.

COMMISSION OF THE EUROPEAN COMMUNITIES. COM (2014) 15 final. Comisión Europea. Comunicación de la Comisión al Parlamento Europeo, al Consejo, al Comité Económico y Social Europeo y al Comité de las Regiones. Un marco estratégico en materia de clima y energía para el periodo 2020-2030. Bruselas, 22.1.2014.

COMMISSION OF THE EUROPEAN COMMUNITIES . COM (2015), 293 final. Comisión Europea. Informe de la Comisión al Parlamento Europeo, al Consejo, al Comité Económico y Social Europeo y al Comité de las Regiones. Informe de Situación en Materia de Energías Renovables. Bruselas, 15.6.2015.

COMMISSION OF THE EUROPEAN COMMUNITIES. COM (2016) 767 final. 2016/0382 (COD). Comisión Europea. Propuesta de Directiva del Parlamento Europeo y del Consejo relativa al fomento del uso de energía procedente de fuentes renovables (refundición). Bruselas, 23.2.2017. 
COMMISSION OF THE EUROPEAN COMMUNITIES. COM (2017) 57 final. Informe d la Comisión al Parlamento Europeo, al Consejo, al Comité Económico y Social Europeo y al Comité de las Regiones. Informe de situación en materia de energías renovables. Bruselas, 1.2.2017.

COUNCIL OF THE EUROPEAN UNION. Presse 138. Proposal on indirect land-use change: Council reaches agreement. Luxembourg, 2014.

DI LUCIA, L. Too difficult to govern? An assessment of the governability of transport biofuels in the EU. Energy policy, 63, 2013.

UE. Dictamen 2009/C 77/12. Dictamen del Comité Económico y Social Europeo sobre la "Propuesta de Directiva del Parlamento Europeo y del Consejo relativa al fomento del uso de energía procedente de fuentes renovables". Diario Oficial de la Unión Europea C 77/43. 31.3.2009.

PARLAMENTO EUROPEU E DO CONSELHO. Directiva (UE) 2015/1513. Parlamento Europeo y el Consejo de 9 de septiembre de 2015 por la que se modifican la Directiva 98/70/CE, relativa a la calidad de la gasolina y el gasóleo, y la Directiva 2009/28/CE, relativa al fomento del uso de energía procedente de fuentes renovables. 15.9.2015.

PARLAMENTO EUROPEU E DO CONSELHO. Directiva 2003/17/CE. Parlamento Europeo y el Consejo de 3 de marzo de 2003 por la que se modifica la Directiva 98/70/CE relativa a la calidad de la gasolina y el gasóleo. Diario Oficial de la Unión Europea. L 76/10. 22.3.2003

PARLAMENTO EUROPEU E DO CONSELHO. Directiva 2003/30/CE del Parlamento Europeo y del Consejo, de 8 de mayo de 2003, relativa al fomento del uso de biocarburantes $\mathrm{u}$ otros combustibles renovables en el transporte. Diario Oficial de la Unión Europea. L-2003-80722.

PARLAMENTO EUROPEU E DO CONSELHO. Directiva 2009/28/ CE. Modifica la Directiva 98/70/CE, relativa a la calidad de la gasolina y el gasóleo, introduciendo la regulación de la sostenibilidad de los biocarburantes. Diario Oficial de la Unión Europea. L 140/88 5.6.2009

PARLAMENTO EUROPEU E DO CONSELHO. Directiva 2009/28/ CE. Parlamento Europeo y el Consejo de 23 de abril de 2009 relativa al fomento del uso de energía procedente de fuentes renovables y por la que se modifican y se derogan las Directivas 2001/77/CE y 2003/30/CE. Diario Oficial de la Unión Europea. L 140/16. 5.6.2009 
PARLAMENTO EUROPEU E DO CONSELHO. Directiva 98/70/CE. Parlamento Europeo y el Consejo de 13 de octubre de 1998 relativa a la calidad de la gasolina y el gasóleo y por la que se modifica la Directiva 93/12/CEE del Consejo. Diario Oficial de las Comunidades Europeas. L 350/58. 28.12.98.

EIA. Base de datos de la Administración de Información de Energía de los Estados Unidos. International Energy Statistics. 2017. Disponiblelenlhttp:// www.eia.gov/cfapps/ipdbproject/iedindex3.fm?tid=79\&pid=79\&aid=1\&c $\mathrm{id}=\mathrm{r} 2$,\&syid=2000\&eyid=2012\&unit=TBPD. Acceso en: 12 jun. 2019.

EUROBSERV'ER. Biofuels Barometer. Systèmes Solaires le Journal des énergies renouvelables N. 2161-2013. Informe del consorcio Eurobserv'er (UE), 2013.

EUROBSERV'ER . Biofuels Barometer, Julio 2015. Informe del consorcio Eurobserv'er (UE), 2015.

EUROSTAT. Base de datos de la Comisión Europea. 2017. Disponible en http://ec.europa.eu/eurostat/data/database. Acceso en: 10 jun. 2019

FAO (2013). Biofuel development should not compromise food security, says CFS. Artículo de noticia en línea.

GRINSVEN, A. Towards a sustainable biofuel trade an analysis of trade barriers and WTO-consistency of certification schemes for biofuels as currently being developed in the EU (Tesis doctoral). Wageningen University, Wageningen (Países Bajos), 2009. Disponible en https://www. wur.nl/upload_mm/d/6/b/04998605-ac22-408c-a3d4-deedb126c6a6 MScthesisTowardsasustainablebiofueltrade.pdf. Acceso en: 16 de junio de 2017.

GUPTA, V. K.; TUOHY, M. G. Biofuel technologies. Recent Developments. Springer, 2013.

KOIZUMI, T. Biofuels and food security. Renewable and Sustainable Energy Reviews, v.52, p. 829-841, 2015.

LONDO, H. M.; DEURWAARDER, E. P.; VAN THUIJL, E. Review of EU Biofuels Directive Public Consultation Exercise. Summary of the responses. Amsterdam: Energy Research Centre of the Netherlands, 2006.

LYDGATE, E. B. The EU, the WTO and Indirect Land-Use Change. Journal of World Trade, vol. 47, n. 1, p. 159-186, 2013. 
ONU. Transformar nuestro mundo: la Agenda 2030 para el Desarrollo Sostenible, 2015. Disponible en: https:/unctad.org/meetings/es/ SessionalDocuments/ares70d1_es.pdf. Acceso en: 3 de jan. 2019.

ONU. Framework Convention on Climate Change. Protocolo de Kioto, 2017. Disponible en http://unfccc.int/portal_espanol/informacion_basica/ protocolo_de_kyoto/items/6215.php. Acceso en: 6 de abr. 2019

PACHAURI, R. K. et al. Cambio climático 2014 Informe de sintesis. Informe del Grupo Intergubernamental de Expertos sobre el Cambio Climático. IPCC, 2014.

PORTOCARRERO, I. R.; RIBEIRO, B. E. Problems ahead or the way forward? An analysis of the red directive, certification schemes, WTO agreements and the social sustainability of biofuels. International conference on governing sustainable biofuels: markets, certification and technolog., Copenhagen biofuels research network (COBREN). Copenhague, Dinamarca, 2012.

SÁNCHEZ-MACÍAS, J. I. et al. Desarrollo agroindustrial de biocombustibles en Castilla y León. Consejo Económico y Social de Castilla y León. Colección de Estudios, 2006.

Artículo recibido en: 01/08/2019

Artículo aceptado en: 08/10/2019

\section{Cómo citar este artículo (ABNT):}

PORTOCARRERO, I. R.; FERRAZ, D. A.; OLIVEIRA, L. P. S. Los biocarburantes sostenibles: un análisis del marco regulatorio de la Unión Europea. Veredas do Direito, Belo Horizonte, v. 16, n. 36, p. 67-89, set./ dez. 2019. Disponible en: $<$ http://www.domhelder.edu.br/revista/index. $\mathrm{php} / \mathrm{veredas} /$ article/view/1614>. Acceso en: día de mes de año. 